PEB Échanges, Programme pour la construction et l'équipement de l'éducation 1999/01

\title{
L'attribution du prix de la construction scolaire aux Pays-Bas
} OCDE 


\section{Projets}

\section{L'ATTRIBUTIO N DU PRIX DE LA CONSTRUC- TIO N SCO LAIRE AUX PAYS-BAS}

Le prix de la construction scolaire est attribué tous les deux ans depuis 1992 au conseil d'établissement néerlandais qui s'est révélé capable de faire preuve d'innovation dans la conception architecturale de son établissement tout en respectant le budget dont il dispose. Ce prix, salué comme un moyen de mettre en place une architecture scolaire de qualité aux Pays-Bas, fait connaître les projets couronnés de succès, de sorte que d'autres écoles peuvent tirer parti des innovations conceptuelles et des renseignements communiqués. Sont associés à ce prix : le ministère néerlandais de l'Éducation, de la Culture et des Sciences ; le ministère du Logement, de l'Aménagement urbain et rural, et de la Gestion environnementale ; I'Association des municipalités néerlandaises.

Pour le prix 1998, ont été invités à participer tous les conseils d'établissement qui avaient investi au moins 500000 florins pour construire ou considérablement agrandir ou rénover une école élémentaire entre 1994 et 1997. Le concours était réservé aux établissements primaires car le prix précédent avait été attribué à un établissement de formation pour adulte. Le jury comprenait des architectes, des enseignants, des représentants du gouvernement et un urbaniste. Ils ont évalué les projets en fonction des critères suivants :

- méthode et projet : la méthode employée pour sélectionner l'architecte et les experts-conseils ; l'aptitude du clientà constituer une équipe équilibrée et à gérer le projet; le plan dans son ensemble et la diversité des espaces à la disposition des élèves ; la polyvalence des bâtiments permettant de répondre aux besoins éducatifs actuels et futurs ;

- interprétation architecturale : I'interaction entre des éléments tels que la structure, l'espace, la lumière et le choix des matériaux et leur influence sur l'atmosphère du bâtiment et la qualité des installations ; la mesure selon laquelle l'architecte a réussi à obtenir la participation de ses clients à la conception ;

- construction : I'utilisation de matériaux durables pour que le bâtiment ait la durée maximum de vie ;

- adaptation à l'aménagement urbain et au paysage : l'intégration spatiale du bâtiment dans son quartier ainsi qu'une délimitation claire entre l'école et son environnement ;

- qualité et coût: les dépenses encourues par rapport à la qualité du bâtiment et au budget disponible.
Premier prix : l'École britannique de La Haye

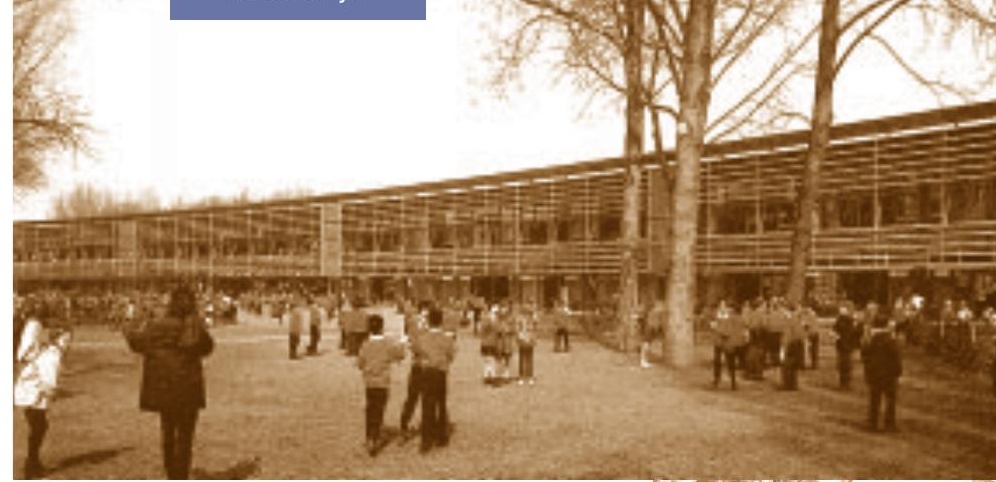

\section{Premier prix}

Le prix de la construction scolaire de 1998 a été attribué à l'École britannique de La Haye, établissement assurant un enseignement élémentaire et secondaire du premier cycle. Certes, cette école a été construite dans le respect du code néerlandais de la construction par une entreprise du bâtiment et un architecte néerlandais, mais le conseif d'établissement n'était pas assujetti aux procédures néerlandaises de prise de décisions concernant la construction de bâtiments scolaires. Cette situation a peut-être donné à l'école britannique une plus grande liberté de manœuvre, mais on a estimé que son coût de construction - 11241955 florins - était comparable à celui indiqué pour les autres établissements présentés.

Le jury a estimé que cette école s'intégrait remarquablement bien dans son environnement. Le bâtiment se trouve sur un site où existaient déjà une salle de sports et un jardin, et le jardin se fond de façon harmonieuse dans le paysage de l'école. Toutefois, l'établissement est isolé au sein de sa localité.

L'école comprend deux principaux bâtiments, I'un rectangulaire, l'autre incurvé, chacun ayant son propre style fonctionnel et architectural. Les salles de classe sont situées dans le bâtiment incurvé donnant sur le jardin et le bâtiment rectangulaire abrite tous les autres services. Les deux bâtiments sont réunis par un couloir spacieux avec une mezzanine d'où I'on peut parfaitement observer les déplacements des élèves.

II s'agit d'un bâtiment véritablement « écologique » construit en matériaux durables. Le bois, le verre, le métal et le béton sont visibles à l'état naturel et utilisés de façon à ne présenter aucun danger pour I'environnement. Des touches de couleur ont été appliquées çà et là.

O n a eu recours dans cet établissement à la régulation climatique naturelle : le refroidissement 


\section{Mentions honorables}

L'une des deux mentions honorables a été attribuée à une école élémentaire M ontessori, l'école De Petteflet, à Tilburg. Cet établissement, d'une superficie hors tout de $1750 \mathrm{~m}^{2}$, a été construit pour un coût de 3485000 florins. La conception de l'école s'inspire de trois principales formes qui jouent un rôle important dans la pédagogie montessorienne:

- le cercle - créé par la tour cylindrique qui abrite les zones réservées au personnel ;

- le triangle - représenté par le bâtiment qui comprend la plupart des salles de classe, celle des premières années étant située au rez-de-chaussée et celle des classes supérieures en haut ;

- le carré - formé par la section comprenant les salles de classe des niveaux intermédiaires et les aires intérieures de jeux.

Le jury a estimé que les formes géométriques étaient au fond une bonne idée bien que parfois à l'origine d'une certaine exiguïté. Chacune de ces sections a sa propre gamme de couleurs et ses propres matériaux de construction. On a conservé aux matériaux leur couleur naturelle, ce qui permet aux enfants de les reconnaître facilement.

Des espaces réservés au travail personnel sont aménagés à l'intérieur et à l'extérieur des salles de classe, et des zones d'étude sont installées dans les niches de fenêtres pour les élèves plus âgés. Les plus jeunes ont leur propre salle de jeux à côté de leurs classes.

L'école est située dans une zone d'aménagement urbain entre différents quartiers. La zone comprend surtout des bâtiments industriels et des terrains de sport, et des routes la séparent des zones d'habitation.

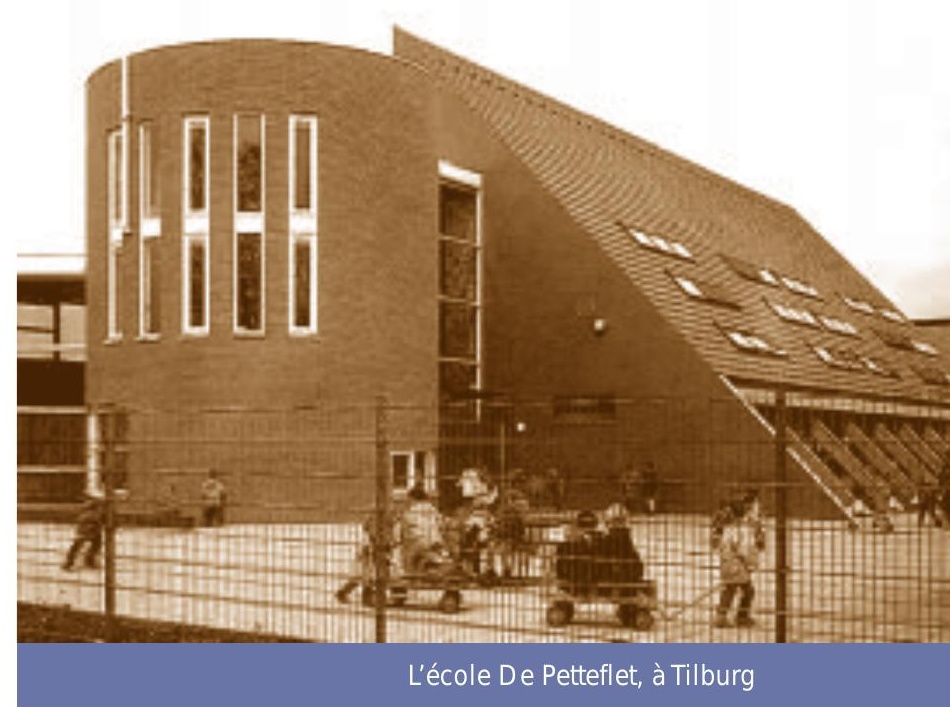


Là encore, l'inspiration architectural e et les objectifs éducatifs indiqués par le client ont de toute évidence pu être conciliés grâce à un dialogue constructif.

Une seconde mention honorable a été attribuée à I'école primaire Het Spectrum, située à Almere. Cet établissement a une superficie hors tout de $2520 \mathrm{~m}^{2}$ et a coûté 4600000 florins.

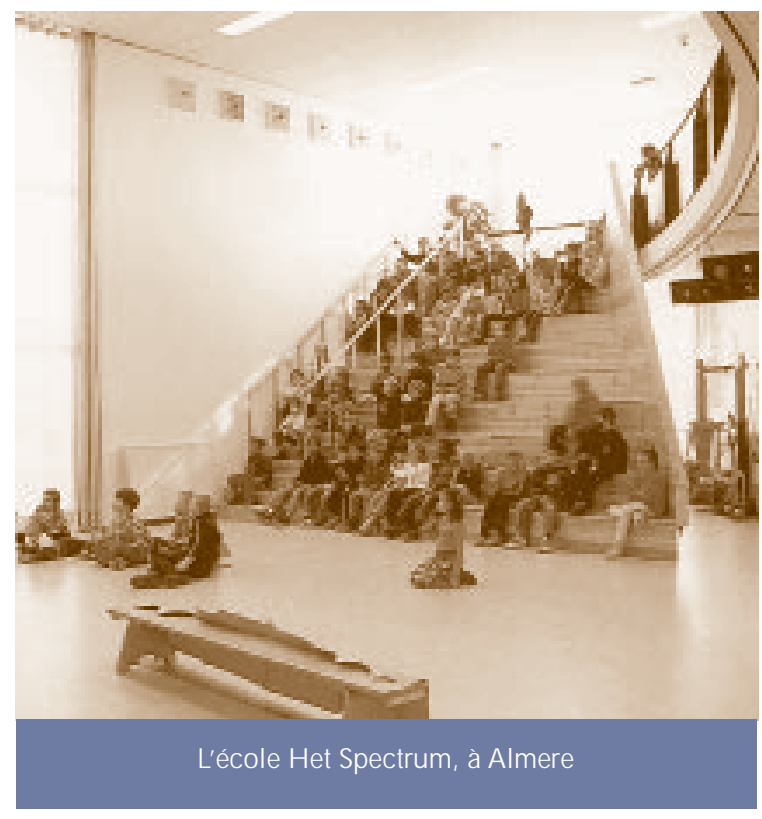

Cet établissement présente une structure claire et de grands volumes dont, selon le jury, les enfants se rappelleront pendant longtemps. II abrite une grande diversité d'espaces, chaque salle de classe ayant une forme différente. Tout comme l'école De Petteflet, les salles de classe destinées aux premières années, aux années intermédiaires et aux années supérieures sont regroupées séparément, chaque niveau se distinguant par son propre camaïeu de couleurs. Plusieurs éléments dans l'école donnent une impression de gaieté : la superbe salle de jeux ou encore le mur d'improvisation théâtrale dans la salle commune. Le jury a constaté que le client avait donné des instructions claires lors du processus de construction et que I'architecte avait eu sincèrement à cœur de refléter le monde des enfants, ce qui a conduit à la construction d'un bâtiment modulable, propice aux jeux et à la stimulation.

\section{Conclusions générales du jury}

Le jury a estimé que parmi les 41 établissements participants, beaucoup étaient dotés d'une construction trop légère, exigeant la mise en place d'installations supplémentaires de chauffage, de climatisation et de ventilation afin d'assurer une atmosphère interne adéquate, d'où une augmentation des frais de fonctionnement. Le jury a signalé que la plupart des conceptions ne faisaient une place suffisante ni aux technologies de l'information et des communications ni aux équipements nécessaires pour mettre en pratique les nouvelles pédagogies. La cour de récréation est aussi souvent négligée ; il en résulte que des écoles attrayantes sont situées dans des cadres médiocres et qu'aucune délimitation claire ne les sépare de leur environnement immédiat. Parmi les autres erreurs couramment constatées, on peut citer les escaliers conçus avec des marches trop élevées ou construits en acier, rendant par là même plus dangereuses les chutes inévitables.

D ans la conception d'un bâtiment scolaire, tout l'art est de créer un environnement dont la structure, les stimulants visuels et l'acoustique contribuent au développement harmonieux des élèves. Dans la mesure où dans le primaire les élèves sont de plus en plus incités à explorer seuls leur environnement, les bâtiments devraient permettre une liberté de mouvement tant à l'intérieur qu'à l'extérieur des classes, prévoir un nombre suffisant d'espaces de travail personnel où les enfants se sentent à l'aise, et comprendre des zones supplémentaires pour toutes sortes d'activités.

Il est important qu'avant la conception, les expertsconseils, les architectes et les clients se mettent d'accord sur les principaux besoins. Ensuite, chaque partie doit évaluer, et, si nécessaire, revoir le projet. S'il est vrai que l'architecte est responsable de la qualité des structures du bâtiment, le programme d'enseignement de l'école n'en demeure pas moins la toute première priorité. Pour que la conception du bâtiment en tienne compte, il est impératif de décrire dans le détail les pratiques pédagogiques, les méthodes de travail et les situations éducatives que l'établissement scolaire veut créer.

O n peut réduire les risques financiers en concevant des bâtiments modulables, ce qui augmente les possibilités d'utilisation (mais il convient d'éviter une trop grande polyvalence qui peut faire de l'école une construction sans originalité et sans identité propre).

Le jury est arrivé à la conclusion qu'à l'avenir les investisseurs seraient bien avisés d'accepter le surcroît d'investissements financiers qu'exige souvent la construction de bâtiments scolaires bien conçus.

Un ouvrage de 48 pages en anglais et en néerlandais décrit dans le menu détail le mécanisme d'attribution de ces prix et comprend des photographies ainsi que les plans des neuf établissements finalistes. Des exemplaires de «School Building Prize 1998 » peuvent être obtenus auprès de : ICS Adviseurs

B.P. 282

2800 AG Gouda, Pays-Bas

tél. : 31182 575200, télécopie : 31182575201. 